\title{
Sequence analyses of fimbriae subunit FimA proteins on Actinomyces naeslundii genospecies I and 2 and Actinomyces odontolyticus with variant carbohydrate binding specificities Mirva Drobni ${ }^{\dagger}$, Kristina Hallberg ${ }^{\dagger}$, Ulla Öhman, Anna Birve, Karina Persson, Ingegerd Johansson and Nicklas Strömberg*
}

Address: Department of Odontology/Cariology, Umeå University, SE-901 87 Umeå, Sweden

Email: Mirva Drobni - Mirva.Drobni@odont.umu.se; Kristina Hallberg - Kristina.Hallberg@odontologi.gu.se; Ulla Öhman - Ulla.ohman@odont.umu.se; Anna Birve - Anna.Birve@odont.umu.se; Karina Persson - Karina.Persson@odont.umu.se; Ingegerd Johansson - Ingegerd.Johansson@odont.umu.se; Nicklas Strömberg* - Nicklas.Stromberg@odont.umu.se

* Corresponding author †Equal contributors

Published: 10 May 2006

BMC Microbiology 2006, 6:43 doi:10.1186/147/-2180-6-43

This article is available from: http://www.biomedcentral.com/I47I-2/80/6/43

(C) 2006 Drobni et al; licensee BioMed Central Ltd.

This is an Open Access article distributed under the terms of the Creative Commons Attribution License (http://creativecommons.org/licenses/by/2.0), which permits unrestricted use, distribution, and reproduction in any medium, provided the original work is properly cited.

\begin{abstract}
Background: Actinomyces naeslundii genospecies I and 2 express type-2 fimbriae (FimA subunit polymers) with variant $\mathrm{Gal} \beta$ binding specificities and Actinomyces odontolyticus a sialic acid specificity to colonize different oral surfaces. However, the fimbrial nature of the sialic acid binding property and sequence information about FimA proteins from multiple strains are lacking.
\end{abstract}

Results: Here we have sequenced fimA genes from strains of A.naeslundii genospecies I $(n=4)$ and genospecies $2(n=4)$, both of which harboured variant Gal $\beta$-dependent hemagglutination (HA) types, and from A.odontolyticus PK984 with a sialic acid-dependent HA pattern. Three unique subtypes of FimA proteins with $63.8-66.4 \%$ sequence identity were present in strains of $A$. naes/undii genospecies $I$ and 2 and $A$. odontolyticus. The generally high FimA sequence identity $(>97.2 \%)$ within a genospecies revealed species specific sequences or segments that coincided with binding specificity. All three FimA protein variants contained a signal peptide, pilin motif, E box, proline-rich segment and an LPXTG sorting motif among other conserved segments for secretion, assembly and sorting of fimbrial proteins. The highly conserved pilin, E box and LPXTG motifs are present in fimbriae proteins from other Gram-positive bacteria. Moreover, only strains of genospecies I were agglutinated with type-2 fimbriae antisera derived from $A$. naeslundii genospecies I strain 12104, emphasizing that the overall folding of FimA may generate different functionalities. Western blot analyses with FimA antisera revealed monomers and oligomers of FimA in whole cell protein extracts and a purified recombinant FimA preparation, indicating a sortase-independent oligomerization of FimA.

Conclusion: The genus Actinomyces involves a diversity of unique FimA proteins with conserved pilin, E box and LPXTG motifs, depending on subspecies and associated binding specificity. In addition, a sortase independent oligomerization of FimA subunit proteins in solution was indicated. 


\section{Background}

Streptococcus and Actinomyces species, e.g. A. naeslundii genospecies 1 and 2 and $A$. odontolyticus (referred to as species), constitute a large portion of the commensal microflora on oral surfaces $[1,2]$. While $A$. odontolyticus dominates at tongue surfaces, $A$. naeslundii genospecies 1 and 2 colonize plaque and buccal surfaces but with different patterns $[1,3,4]$. Moreover, Actimomyces species have been implicated in dental caries, periodontitis and other infections [5-8].

Besides adhesion to salivary pellicles and oral epithelial surfaces $[9,10]$, actinomycetes and streptococci participate in inter- and intra generic coaggregations, as defined by the Actinomyces-Streptococcus coaggregation groups $\mathrm{A}$ to $\mathrm{F}$ for A. naeslundii genospecies 1 (i.e. groups $\mathrm{B}, \mathrm{C}$ and $\mathrm{D}$ ), genospecies 2 (i.e. groups $\mathrm{A}$ and $\mathrm{F}$ ) and $A$. odontolyticus (i.e. group E) $[9,11]$. To participate in these adhesive interactions, Actinomyces naeslundii genospecies 1 and 2 express two antigenically different fimbriae, type- 1 and type- 2 [12-14]. Type-1 fimbriae bind to acidic proline-rich proteins and statherin in salivary pellicles on teeth $[13,15]$. Type- 2 fimbriae contribute to adhesion and colonisation [13] by binding to Gal $\beta$ structures (i.e. $\beta$-linked galactose or galactosamine) [16] in salivary pellicles [17], streptococci [18], oral epithelial cells $[19,20]$ and to polymorphonuclear leucocytes [21]. Both genospecies 1 and 2 express type-2 fimbriae but with variant Gal $\beta$ binding specificities $[14,20]$, and each genospecies exhibits at least two types of Gal $\beta$-based hemagglutination patterns [1].

The major subunit genes of type- 2 and type- 1 fimbriae, fimA and fimP, respectively, have been cloned and sequenced from $A$. naeslundii genospecies 1 (strain 12104) and 2 (strain T14V) [22-26]. The deduced FimA and FimP subunit proteins are 534 and 533 amino acid proteins, respectively, with $34 \%$ amino acid identity. FimA and FimP contain seven conserved proline-containing regions involved in folding of the two proteins and an LPXTG sorting signal followed by a N-terminal membrane spanning domain [25]. Structurally diverse fimA and fimP genes, as well as species-specific fim $A$ gene segments, have been found for $A$. naeslundii genospecies 1 and 2, and linked to different coaggregation groups and types of Gal $\beta$ - and PRP- related adhesion properties [14,27]. However, the fimA gene has so far only been sequenced from a single strain of both genospecies 1 (12104) and 2 (T14V) $[24,25]$.

A. odontolyticus is a prominent member on the tongue as well as present at supra- and subgingival sites $[1,4]$. The fimbrial structure of $A$. odontolyticus, and host receptors, employed for its adhesion have not been fully investigated. However, inhibition studies show that sialylated carbohydrate structures, such as sialyl Tn and 3' sialyllac- tose structures, serve as a salivary glycoprotein gp-340 receptor for A. odontolyticus strain PK984 [28], which is a reference strain for coaggregation group E. Moreover, hybridization studies [14] have indicated FimA- or FimPrelated adhesins on $A$. odontolyticus, but fimA or fimP genes have not yet been identified or sequenced from $A$. odontolyticus.

The aim of this study was to characterize fimA genes from several strains of $A$. naeslundii genospecies 1 and 2 with variant Gal $\beta$ binding specificities and from a strain of $A$. odontolyticus PK984 with a sialic acid binding specificity.

\section{Results}

\section{A. naeslundii genospecies I and 2 and $A$. odontolyticus PK984 display deviating cell binding properties}

A. naeslundii genospecies 1 and 2 express type- 2 fimbriae with variant types of Gal $\beta$ binding specificity and $A$. odontolyticus express a sialic acid binding specificity potentially related to type-2 fimbriae (Table 1 ). Accordingly, while A. odontolyticus PK984 agglutinated untreated but not sialic acid-depleted red blood cells, A. naeslundii genospecies 1 and 2 (strains 12104 and LY7, respectively) agglutinated only sialic acid depleted cells strongly, due to exposure of penultimate Gal $\beta$-residues (Table 1).

Table I: Type-2 fimbriae subtypes on A. naeslundii genospecies I and 2 and $A$. odontolyticus with different binding properties.

\begin{tabular}{|c|c|c|c|}
\hline & \multicolumn{2}{|c|}{ A. naeslundii } & \multirow[t]{2}{*}{ A. odontolyticus } \\
\hline & gsp I & gsp 2 & \\
\hline & 12104 & LY7 & PK984 \\
\hline Fimbriae type ${ }^{a}$ & $2: 1$ & $2: 2$ & $2: 3$ \\
\hline \multicolumn{4}{|l|}{ Sugar specificity ${ }^{b}$} \\
\hline Gal $\beta$ & $+(G \mathrm{~L})$ & $+(\mathrm{GP})$ & - \\
\hline Sialic acid & - & - & $+(\mathrm{GP})$ \\
\hline \multicolumn{4}{|l|}{ Hemagglutinationc } \\
\hline Human & I & 0 & 4 \\
\hline Human + sialidase & 3 & 4 & l \\
\hline
\end{tabular}

aSubtypes of type-2 fimbriae, types-2:I to 2:3, among Actinomyces species based on different hybridization patterns of strains with fimAderived DNA probes and binding patterns to carbohydrates, red blood cells and bacterial partners [14].

b Saccharide specificity and receptor ligand $(\mathrm{GL}=$ glycolipid, $\mathrm{GP}=$ glycoprotein) for each fimbriae subtype or reference strain $[20,28]$. c Hemagglutination (HA) patterns for each fimbriae subtype or reference strain. Score 0 marks no HA and score 4 strong HA. Similar HA results were obtained with goat and chicken erythrocytes (data not shown). 


\section{Subtypes of FimA proteins in A. naeslundii genospecies I and 2 and $A$. odontolyticus with different binding specificity}

fimA genes were amplified by PCR and sequenced from strains representative for genospecies 1 (12104, Pn-22-E, Pn-6-N, P-5-N), genospecies 2 (T14V, P-1-N, P-1-K, LY7) and for A. odontolyticus (PK984) with different Gal $\beta$ and sialic acid binding properties, respectively. In addition, the A. naeslundii strains were selected to include two types of Gal $\beta$-dependent hemagglutination patterns for each genospecies.

Structurally variant FimA proteins were found in A. naeslundii genospecies 1 and 2 and A. odontolyticus, respectively (Table 2, Fig. 1). The FimA sequence identity between the three species was in the $62.8-66.4 \%$ range, while the sequence identity between strains of the same species was 88.6-99.6 \%. Accordingly, sequence analyses of the FimA proteins, clustered FimA proteins from genospecies 1 and 2 and A. odontolyticus into separate groups, but the two latter species more closely (Fig. 1). In addition, the SpaH fimbriae protein from Corynebacterium diphteriae clustered more closely to FimA than did FimP from A. naeslundii.

\section{Structural features of the novel FimA protein in A. odontolyticus with a sialic acid specificity}

The fimA gene of A.odontolyticus PK984 encodes a 535 amino acid protein (Fig. 2). The FimA protein contains: $i$ ) an $\mathrm{N}$-terminal signal peptide with a signal peptidase cleavage site, ii) a pilin motif for polymerisation of subunit monomers, iii) a proline-rich segment, $i v$ ) an E box motif, $v$ ) an LPXTG sorting motif, and vi) a C-terminal cell membrane spanning domain.

Cleavage of the 535 amino acid FimA protein of A.odontolyticus between residues 32 and 33 would generate a fimbrial subunit protein of 503 residues. This gives a theoretical subunit molecular weight of $52.6 \mathrm{kDa}$.

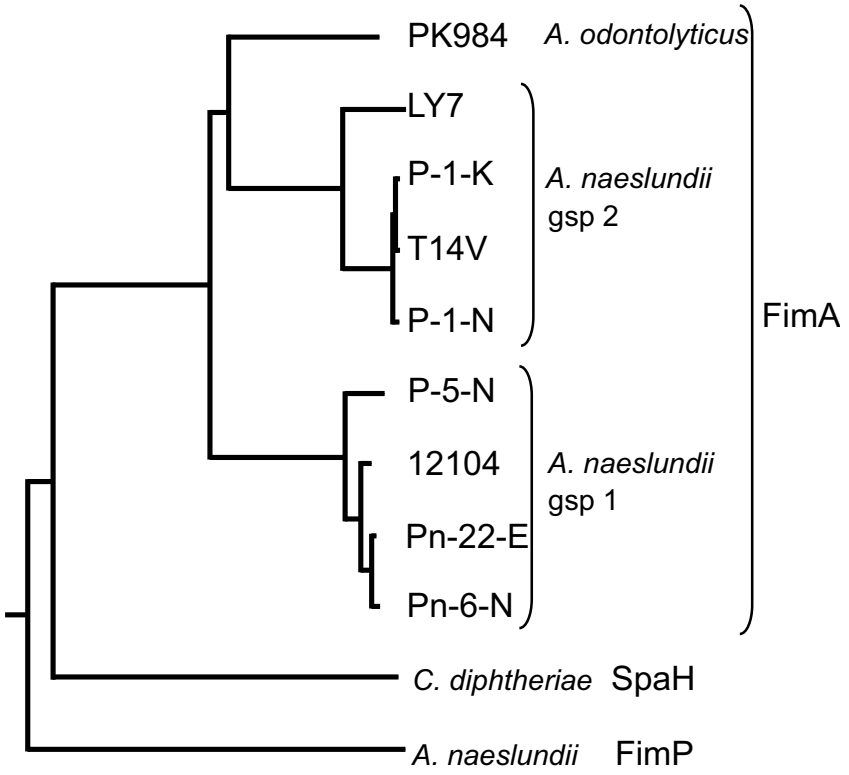

\section{Figure I}

Dendrogram of FimA proteins. Sequence analyses were preformed on FimA proteins from $A$. naeslundii genospecies I (strains 12104, Pn-22-E, Pn-6-N, P-5-N), A. naeslundii genospecies 2 (strains TI4V, P-I-N, P-I-K, LY7) and A. odontolyticus (strain PK984) using Clustal W. The SpaH protein from $C$. diphtheriae and FimP from A. naeslundii LY7 genospecies 2 were included as out-groups.

\section{Species-specific and conserved segments in FimA proteins from $A$. naeslundii genospecies $I$ and 2 and $A$. odontolyticus}

The FimA proteins from strains of $A$. naeslundii genospecies $1(n=4)$ and $2(n=4)$ and $A$. odontolyticus $(n=1)$ were compared for FimA sequences or segments conserved between the species or unique to each species (Fig. 3A). The generally high sequence identity (>97.2\%) within a genospecies (Table 3 ) revealed species specific sequences or segments that coincided with binding specificity.

Among sequences conserved between FimA proteins are the N-terminal signal peptide, pilin motif (WXYDVXVYPKN), proline-rich segment (PPXPXXPPXXPXNPP), E box (LVETKAPXGX) and LPXTG sorting motif (LPLTG),

Table 2: Inter- and intra-species sequence identity of FimA subunit proteins ${ }^{a}$

\begin{tabular}{|c|c|c|c|}
\hline \multirow[b]{2}{*}{ Strains ${ }^{b}$} & \multicolumn{2}{|c|}{ A. naeslundii } & \multirow{2}{*}{$\begin{array}{l}\text { A. odontolyticus } \\
\text { PK984 FimA 2:3 }\end{array}$} \\
\hline & Genospecies I I2104 FimA 2:I & Genospecies 2 TI4V FimA 2:2 & \\
\hline A. naeslundii gsp I $(n=4)$ & $93.8-97.6$ & $62.8-64.1$ & $63.7-65.2$ \\
\hline A. naeslundii gsp $2(n=4)$ & $63.8-64.4$ & $88.6-99.6$ & $64.9-66.4$ \\
\hline
\end{tabular}

a Sequence identity (\%) of strains as compared to strains $12104, \mathrm{TI} \mathrm{V}$ and PK984. FimA 2:1 to 2:3 denotes FimA proteins associated with the type2 fimbrial subtypes 2:I to 2:3 with different binding patterns to carbohydrates, red blood cells and bacterial partners $[14,20,28]$.

b Genospecies I strains were I2I04, Pn-22-E, Pn-6-N and P-5-N, and genospecies 2 strains were TI4V, P-I-N, P-I-K and LY7. 
A.n 12104

A. O PK984

A. n 12104

A. 0 PK984

A.n 12104

A. 0 PK984

A. $n 12104$

A. $O$ PK984

A.n 12104

A. O PK984

A.n 12104

A. O PK984

A. 12104

A. O PK984

A.n 12104

A. 0 PK984

A. n 12104

A. 0 PK 984

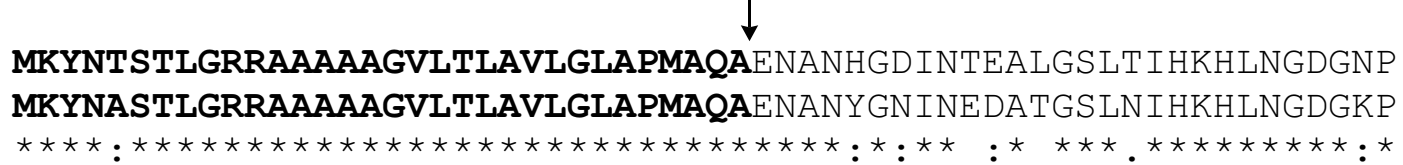

IGAPDGTASNDDGKGAPVSGVQFTAYEINGIDLKTSEGWAKVNALTNTGAIPDNACANPG IGNADGTEVPNANKGAPVKGVVFTAYPITDINLKKSEDWTKLGALTAPGAIPDSACANPA

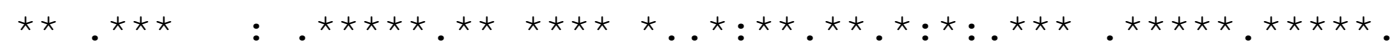

QPTLPNYTERSSRVSGDTDRDGEAKIESLPVKAYLVCETKTPGNIVQKAKPFVVTI PHPN APALAGHTLGSGMPSSETDDQGLAKI SDMSVKAYLVCETKAPGNIVQKAKPFVVT I PHPN

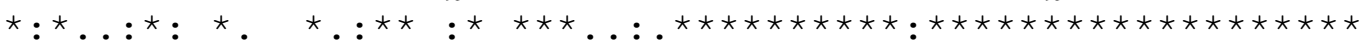

TAAKADGTWLYDVHVYPKNEKIEVAKTIEDQRNNGYIVGSKVRFPVSSTLPKLDDNSYYK TAKDQAGNWIYDVHVYPKNEKTEVTKTIEDQKANGYAVGSKVRFPVSSTLPKLDDGAHYK

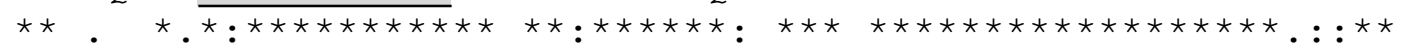

YYQFKDTLDNRLKQVTATDVTLGGTRLDEGTDYTLGTDGQTVTVTFNQNGLSKLKGNPGQ YFQLKDTLDANLTGVTAKDVTLDG-APMEASDYEVKTTGQTVTVTFTKAGLAKLKAAAGK

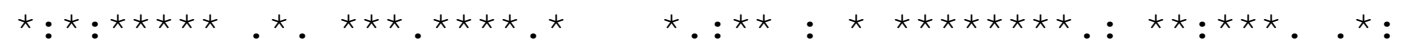

KLQAVFEGVVSEVGDGS INNTAQLISDTTYAEQPPAPETPPANPDNPPTTEQVTSKWGDL KVQAIFEGTVAS IGNGNIKNTAQLISDTIYASTPPEPTEPPTDPDNPPTSDEVTTTWGDL

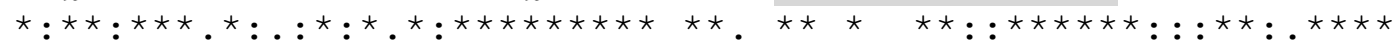

TIKKVDGNDRSGDKDGLKGAEFQIYKAKDAYADTCSPEADGQPLTINGESTFTTGEGGTI TIKKVDSHDKGASKAGLKGAEFQLFKAQKAYDDTCTKEKEGAPIAVDGKTTLTTDENGVV

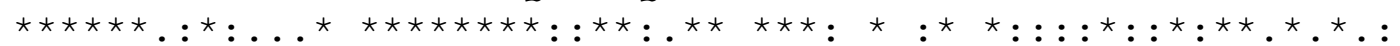

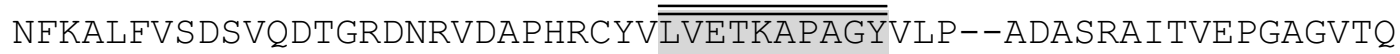
NIKGLFVSDSVAGADRDNKVGATSRCYVLVETKAPTGFVLPSGDDAVTAVKIQAGAVTTD

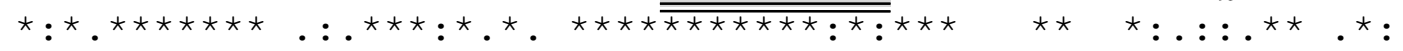

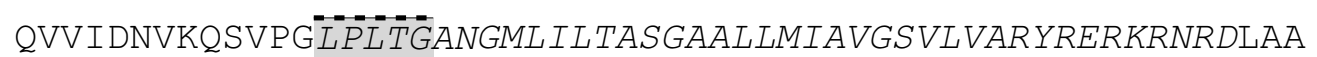
NVTVENTKQAVPGLPLTGANGMLILTASGASLLMIAVGSVLVARYRERKHSANLAA

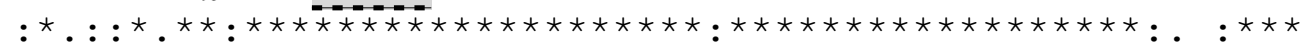

\section{Figure 2}

Sequence of FimA from A. odontolyticus strain PK984. The PK984 FimA sequence was compared to FimA from A. naeslundii genospecies I strain 12104. Identical (*), conserved (:), and similar (.) amino acids are indicated. Marked are the N-terminal signal peptide (bold typeface) and peptidase cleavage site (arrow), pilin motif (grey box lined with single line), a proline-rich region (grey box), E box (grey box lined with double lines), and an LPXTG motif (grey box lined with dashed line). The motifs/ domains are also present in $A$. naeslundii genospecies 2 (not shown).

two additional proline-containing segments, as well as four other segments with high sequence identity (Fig. 3A). The pilin, E-box and LPXTG motifs displayed 80 to $100 \%$ sequence identity for FimA proteins present in A.odontolyticus and A. naeslundii genospecies 1 and 2 (Fig. 3B).
Multiple FimA segments showed a low sequence identity between the species.

Apart from the sequences unique to and conserved within each species, we could not identify or link any distinct motif, sequence or substitution to the different binding or 


\section{A}

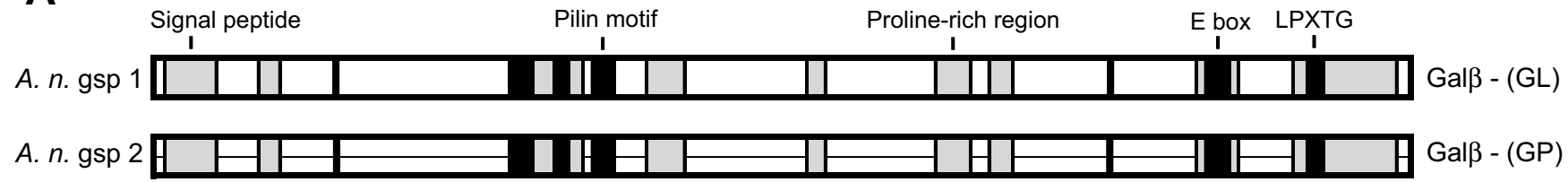

A. 0 .

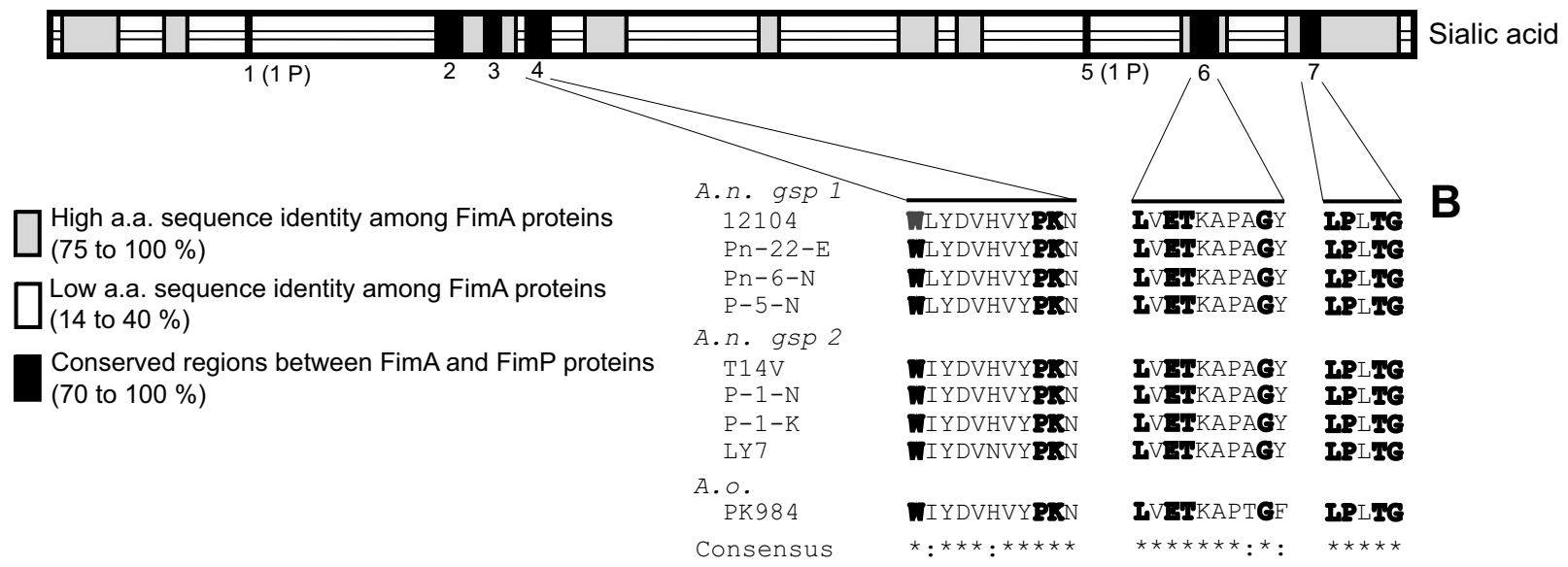

Figure 3

Comparison of FimA protein sequences from A. naeslundiigenospecies I and 2 and $A$. odontolyticus with different binding properties. A) Shown are conserved regions between FimA and FimP proteins (black boxes) and regions with high (grey boxes) or low (white boxes) sequence identity between FimA proteins. The low FimA amino acid (a.a.) sequence identity regions (unlined, double and triple lined white boxes) are species-specific due to a high strain-to-strain sequence identity for each species. High FimA sequence identity domains include the $\mathrm{N}$-terminal signal peptide, pilin motif, proline-rich region, E box, and LPXTG motif (motifs are marked and the seven conserved proline-containing domains identified by Yeung and Cisar are numbered). The proline-containing domains I and 5 of FimA display only a conserved proline residue, in contrast to FimP, in which the entire seven proline-containing domains are conserved. The FimA proteins from genospecies I and 2 and A. odontolyticus contains 32,33 and 32 amino acid signal peptides and 534, 535 and 535 amino acid proteins, respectively. B) Alignment of the pilin, E-box and LPXTG motifs present in FimA from different strains. Postulated key determinants are in bold $[29,39]$.

hem-agglutination types between species or strains. However, the genospecies 1 strain $\mathrm{P}-5-\mathrm{N}$ and genospecies 2 strain LY7 with somewhat lower intra-species sequence identities, $93.8 \%$ and $88.6 \%$, showed unique hemagglutination or coaggregation patterns, respectively (Table 3 ).

Structural comparison of FimA and FimP proteins from $A$. naeslundii, $A$. odontolyticus and $A$. viscosus

The FimP (from $A$. naeslundii genospecies $2, \mathrm{n}=2$, and $A$. viscosus, $\mathrm{n}=1$ ) and FimA protein families contained conserved N-terminal signal peptides and C-terminal membrane spanning segments, but with low sequence identity to each other ( $24 \%$ ) (Fig 3A). High identity sequences in both FimA and FimP are the proline-containing domains 2, 3, 4 (pilin motif), 6 (E box) and 7 (LPXTG). In contrast, the proline-containing regions 1 and 5 are conserved domains in FimP but show only conserved proline residues in FimA.
FimA from $A$. naeslundii genospecies $I$ and 2 and $A$. odontolyticus display different antigenic properties

Strains of $A$. naeslundii genospecies 1 and 2 and A. odontolyticus were tested for reactivity with antisera R70-3 specific to FimA from type- 2 fimbriae of genospecies 1 strain 12104 using whole cell agglutination and Western blot analyses with whole cell proteins (Fig. 4). While all strains of A. naeslundii genospecies 1, except for strain Pn-6-N, were agglutinated by the antisera, neither strains of genospecies 2 nor A. odontolyticus PK984 were agglutinated (Fig. 4A). Thus, the native FimA protein variants possess different antigenic properties and potentially different overall folding patterns.

Moreover, only genospecies 1 strains possessed positive FimA protein bands in Western blot analyses with type 2 fimbriae specific antisera(Fig. 4). Besides the FimA monomer (no. 1) and polymers (no. 3) detected in all genospecies 1 strains, di- to oligomers of FimA (no.2) were also detactable, suggesting either oligomerization of FimA 
Table 3: Sequence identity of FimA proteins within A. naeslundii genospecies I and 2 and associated binding properties.

\begin{tabular}{|c|c|c|c|c|c|c|c|c|}
\hline \multirow[b]{2}{*}{ Strains } & & \multirow{2}{*}{$\begin{array}{c}\text { Sequence } \\
\text { identity }(\%)^{\mathrm{a}}\end{array}$} & \multicolumn{4}{|c|}{ HA pattern ${ }^{b}$} & \multirow[t]{2}{*}{ HA typec } & \multirow[t]{2}{*}{ COG group } \\
\hline & & & Human $(+\mathrm{Na})$ & Porcine & Human & Horse & & \\
\hline \multicolumn{9}{|c|}{ A. naeslundii gsp I } \\
\hline & 12104 & 100 & $1 / 8$ & $1 / 4$ & $\mathrm{I} / 2$ & $1 / 8$ & $\mathrm{a}$ & B \\
\hline & $P n-22-E$ & 97.6 & $1 / 32$ & $1 / 16$ & $\mathrm{I} / 4$ & $1 / 8$ & $\mathrm{a}$ & \\
\hline & Pn-6-N & 97.2 & $1 / 32$ & $1 / 16$ & $1 / 2$ & $1 / 32$ & a & \\
\hline & P-5-N & 93.8 & $1 / 16$ & $1 / 4$ & 0 & 0 & b & \\
\hline \multicolumn{9}{|c|}{ A. naeslundii gsp 2} \\
\hline & TI4V & 100 & $1 / 16$ & 0 & 0 & 0 & a & $A$ \\
\hline & P-I-N & 99.3 & $1 / 16$ & 0 & 0 & 0 & $\mathrm{a}$ & \\
\hline & P-I-K & 99.6 & $1 / 32$ & $1 / 16$ & 0 & $1 / 4$ & b & \\
\hline & LY7 & 88.6 & $1 / 32$ & $1 / 16$ & 0 & $\mathrm{I} / 4$ & $b$ & $\mathrm{~F}$ \\
\hline
\end{tabular}

aAmino acid sequence identity (\%) within each genospecies as compared to strains 12104 or TI4V, respectively.

bHemagglutination (HA) of neuraminidase treated human and untreated porcine, human and horse red blood cells by reciprocal dilutions of bacterial cell suspensions. The lowest dilution with visible HA is given. 0 marks no agglutination even at the highest dilution.

$c a$ and $b$ denotes the two hemagglutination patterns observed in each genospecies.

dCoaggregation properties as defined by coaggregation groups (COG) A to F [27]

subunits or the presence of polymeric fragments of covalently tathered FimA subunits.

\section{Oligomerization of recombinant FimA protein}

To further explore the possible oligomerization of FimA in solution, we expressed and purified the FimA protein from A. naeslundii strain 12104 as a recombinant protein and analysed its ability to oligomerize by gel electrophoresis and Western blot analyses using FimA specific antisera (Fig 4C). Gels of the recombinant FimA protein revealed monomers (no. 1) and oligomers (no. 2) of FimA, as confirmed by Western blotting, verifying the possibility of FimA di- to oligomerization in solution but dependence on whole cells for fimbriae polymerization.

\section{Discussion}

The present study shows three unique subgroups of FimA proteins present in A. naeslundii genospecies 1 and 2 and A. odontolyticus with different glycoconjugate receptors. It therefore supports our hypothesis that commensal micro organisms, like the genus Actinomyces, exhibit complex and divergent mosaics of adhesin molecules related to species or subpopulations with different tropism and ecological niches. Notably, A. naeslundii genospecies 1 and 2 and A. odontolyticus are members of coaggregation groups $\mathrm{A} / \mathrm{F}, \mathrm{B} / \mathrm{C} / \mathrm{D}$ and $\mathrm{E}$, respectively, and differ in a variety of type-1, type-2 and other adhesive properties [14]. The FimA protein, which contains both sequences unique to and conserved between the species, may have evolved to match specific niches in supra- or subgingival plaque or in buccal or tongue epithelial tissues. The novel FimA protein from A. odontolyticus strain PK984, a reference strain for Actinomyces-Streptococcus coaggregations typical of sub- gingival plaque, may mediate coaggregations or adhesive interactions typical of subgingival plaque. Strains of $A$. odontolyticus from the tongue display FimA and FimP hybridization patterns somewhat different from that observed for strain PK984 [14].

All three FimA protein variants contained a pilin motif for polymerization, E box for associated proteins and LPXTG motif for cell surface sorting and anchoring. The pilin, E box and LPXTG motifs were highly conserved among the three species, and present among the proline-containing domains suggested by Yeung and Cisar to account for folding or intermolecular interactions of FimA and FimP proteins [25]. Interestingly, the pilin, E box and LPXTG motifs are present in fimbriae proteins from $C$. diphtheriae, which have been used to express type- 2 fimbriae from $A$. naeslundii, and in various other Gram-positive bacteria expressing pili-like structures $[29,30]$. This suggests that Gram-positive bacteria may have evolved related proteins and pathways for pili formation and function [31]. Moreover, the serologically different properties of the FimA proteins present in genospecies 1 and 2 and A. odontolyticus could imply that the overall folding of the FimA protein could form different functionalities or binding specificities. Actually, the variant FimA proteins did not cross-react with antisera despite many conserved motifs, which consequently may be hidden within the subunit or by intermolecular interactions. Apart from the sequences unique to each species, we could not link any FimA motifs or substitutions to the species-specific binding specificities or variant HA patterns of each genospecies. However, receptor-binding FimA subunit domains or tip-localised adhesins other than the FimA subunit remains to be iden- 


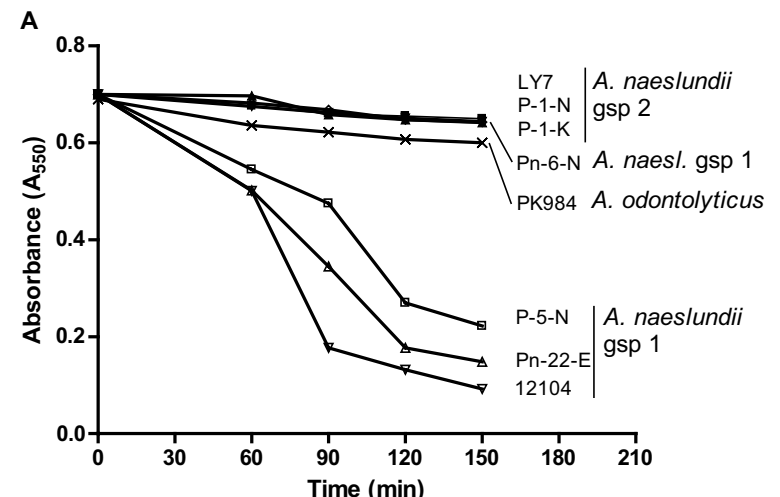

B

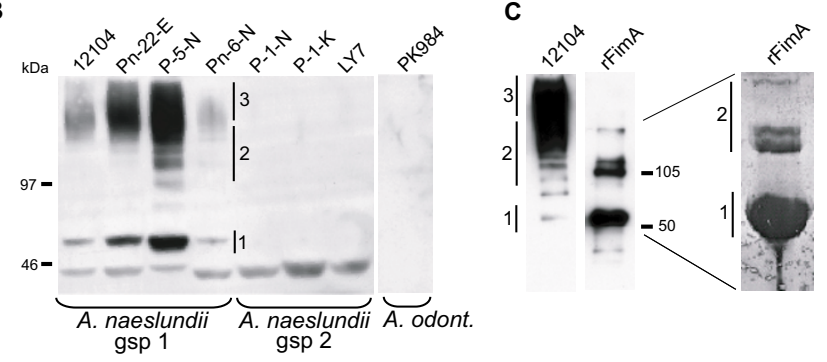

Figure 4

Antigenic properties and oligomerization of native and recombinant FimA. A) Whole cell agglutination by type-2 fimbriae specific antisera (derived from genospecies I ATCC 12 104) of A. naeslundii genospecies I (strains I2 I04, Pn-22-E, P-5-N) but neither of genospecies 2 (strains LY7, P$\mathrm{I}-\mathrm{N}, \mathrm{P}-\mathrm{I}-\mathrm{K}$ ) nor of $A$. odontolyticus (strain PK984). Experiments were repeated three times with similar results. B) Western blots of whole cell proteins from strains of $A$. naeslundii genospecies I and 2 and $A$. odontolyticus PK984 with type-2 fimbriae specific antisera. FimA monomers (no. I), oligomers (no. 2) and polymers (no. 3) are marked. C) Western blots of whole cell proteins from $A$. naeslundii strain 12104 and recombinant FimA from strain 12104, and Coomassie blue-stained gel electrophoresis pattern (to the right) of the mono- to oligomer region of recombinant FimA. Molecular mass markers are indicated.

tified. In this respect the unique presence of a proline-rich segment in FimA but not in FimP proteins is interesting, but of yet unknown biological significance.

Another interesting finding of the present study is the possible sortase-independent oligomerization of FimA in solution. Western blot analyses revealed FimA oligomers in whole cell protein extracts and, more importantly, mono to oligomers of FimA in purified recombinant FimA preparations. Sortase, which is absent in Gram-negative E. coli used for expression of recombinant FimA protein, catalyses the covalent tethering of FimA subunits through pilin and LPXTG motifs when expressed in Grampositive C. diphtheriae [29]. It remains, however, to be determined whether the pilin motif and/or other con- served subunit motifs are involved in this sortase-independent ability of FimA to oligomerize in solution. Finally, it is reasonable to assume that the spontaneous ability of FimA to di- to oligomerize acts in conjunction with sortase in the whole cell-dependent process of fimbriae assembly and polymerization.

The unique and conserved nature of FimA for each Actinomyces species or subspecies reinforces the important role of FimA in selection of ecological niches. Moreover, the highly conserved nature of FimA within a subspecies could also indicate an immunological tolerance to this protein on Actinomyces species that early colonize the oral cavity of infants [3]. The conserved nature of FimA is different to the antigenic variation found in P-fimbriae-associated PapG adhesins on uropathogenic Escherichia coli [32]. Based on its unique and conserved nature, we have previously designed DNA probes from particular FimA segments to distinguish between clinical isolates of genospecies 1 and 2 . We assume that corresponding FimA segments unique to A. odontolyticus could be used in a similar way to generate probes specific to particular receptorbinding subtypes of this organism.

While different Gal $\beta$ specificities of genospecies 1 and 2 target the two species to glycolipid or glycoprotein receptors [20], respectively, A. odontolyticus PK984 recognizes sialic acid residues on glycoproteins. Both glycolipids and glycoproteins are capable of mediating adhesion and intra-cellular signalling by epithelial cells. The host responses mediated by a glycolipid or a glycoprotein receptor may be different, and hypothetically relate to the commensal or pathogenic potential of Actinomyces subtypes. Moreover, the Gal $\beta$ and sialic acid binding specificities may target $A$. naeslundii and $A$. odontolyticus to different bacterial partners, in particular since they belong to different coaggregation groups. In this respect, it is notable that sialidase, which modifies the hemagglutination properties of $A$. naeslundii and $A$. odontolyticus in the opposite way, is produced by Actinomyces and other plaque bacteria [33]. However, whether A. naeslundii or related coaggregation communities use sialidase to compete with A. odontolyticus in vivo is unknown. Finally, A. naeslundii and $A$. odontolyticus are interesting model bacteria for studying the role of fimbriae proteins and their receptors specificities in microbial colonisation and hostmicrobe interactions.

\section{Conclusion}

This report shows three unique subgroups of FimA proteins in A. naeslundii genospecies 1 and 2 and A. odontolyticus, and that each subgroup coincides with a unique carbohydrate binding specificity. All FimA proteins contained a pilin motif for polymerization, E box, and LPXTG motif for cell surface sorting and anchoring. Finally, a sor- 
tase independent oligomerization of FimA subunit proteins in solution was indicated.

\section{Methods}

\section{Bacterial strains and culturing conditions}

The A. naeslundii genospecies 1 strains Pn-22-E (CCUG 34193), Pn-6-N (CCUG 33519), P-5-N (CCUG 33914), ATCC 12104 (CCUG 32832), and A. naeslundii genospecies 2 strains P-1-N (CCUG 33910), P-1-K (CCUG 32838), LY7 (CCUG 33934) and T14V used were generally from the Culture Collection, University of Göteborg (CCUG), Sweden. A.odontolyticus PK984 was provided by Dr. Kolenbrander, National institute of health/NIDCR, Bethesda, USA. The strains were cultured on Columbia-IIagar base plates (Becton Dickinson and Co., Cockeysville, $\mathrm{MD})$, supplemented with $3 \%$ human red blood cells, at $37^{\circ} \mathrm{C}$ in candle jar.

\section{Cloning and sequencing of fimA genes}

Chromosomal DNA was isolated from bacteria and purified as described [14]. Gene segments were amplified by PCR, using standard protocols, by use of primers both inside and outside the fimA open reading frame (primer sequences are available upon request). All fimA PCR fragments were cloned into pGEM-T vectors using T4 DNA ligase (Promega Corp., Madison, WI), except for fimA from A. odontolyticus for which fragments were cloned into pCR 2.1-TOPO vectors using Invitrogen TOPO TA Cloning kit (Invitrogen, Carlsbad, CA).

Plasmid DNA was isolated using the QIAprep ${ }^{\circledast}$ Spin Miniprep Kit (Qiagen GmbH, Hilden, Germany) and the size of DNA inserts were subsequently confirmed by SalII and NcoI or only EcoR1 (Invitrogen) cleavage. Sequencing was done using the Thermo Sequenase radiolabeled terminator cycle sequencing kit (Amersham Life Science, Cleveland, $\mathrm{OH})$, and were performed according to the manufacturer's instruction. The DNA fragments were sequenced in both directions.

\section{Sequence and motif analysis}

Complete open reading frames of fimA nucleotide sequences were analyzed using the Wisconsin Package (version 9.0) from the Genetics Computer Group (GCG, University of Wisconsin, Madison, WI), except for fimA from A. odontolyticus which was analysed with The Molecular Toolkit [34].

Sequence alignments and dendrogram was made using Clustal W (1.83) multiple sequence alignment [35].

Signal peptide, LPXTG motif and proline-rich region were analyzed using bioinformatics tools [36-38].

\section{Nucleotide sequence accession numbers}

The GenBank accession numbers for genes presented in this paper are: for fimA: strain Pn-22-E [GenBank: DQ425098], strain Pn-6-N [GenBank: DQ425100], strain P-5-N [GenBank: DQ425097], strain P-1-N [GenBank: DQ425102], strain P-1-K [GenBank: DQ425099], strain LY7 [GenBank: DQ425101], strain ATCC 12104 [GenBank: $\underline{M}$ 21976], strain T14V [GenBank: $\underline{\text { AF019629] }}$, and strain PK984 [GenBank: DQ425103]; for fimP; strain P-1K [GenBank: AF107019], strain LY7 [GenBank: AF10720], A. viscosus 19246 [GenBank: M 21976]; for SpaH: C. diphteriae NCTC 13129 [GenBank: NP 940533].

\section{Agglutination of Actinomyces by antisera}

Agglutination of Actinomyces cells by rabbit antiserum R70-3 specific for type-2 fimbriae of A. naeslundii strain ATCC 12104 and pre-immunization serum R70-0 was a gift from Dr. Cisar, National institute of health/NIDCR, Bethesda, USA. Antisera $(1 \mu \mathrm{ml})$ was added to Actinomyces cells $\left(1 \mathrm{ml}, \mathrm{OD}_{550}=0.7\right)$ suspended in coaggregation buffer $\left(150 \mathrm{mM} \mathrm{NaCl}, 0.1 \mathrm{mM} \mathrm{CaCl}_{2}, 0.1 \mathrm{mM} \mathrm{MgCl}_{2}, 1.0\right.$ $\mathrm{mM}$ Tris $\mathrm{pH} 8.0$ and $0.02 \%$ sodium azide). After incubation for one hour at room temperature on slow rotation, agglutination was measured by recording the optical density at $550 \mathrm{~nm}$ for $90 \mathrm{~min}$, giving a total agglutination time of $150 \mathrm{~min}$.

Cloning, expression and purification of recombinant FimA The fimA gene was PCR-amplified from genomic DNA from strain 12104 and cloned into the expression vector pETM11 (EMBL, Hamburg, Germany). The resulting construct encodes a protein (recombinant FimA or rFimA) with an N-terminal hexa-histidine tag, an 18 residue long linker and the FimA protein excluding the $\mathrm{N}$-terminal signal sequence and the C-terminal transmembrane helix. $E$. coli BL21 (DE3) (Novagen, Madison, WI) was transformed with the pETM11-rFimA construct and grown at $37^{\circ} \mathrm{C}$ to optical density $\left(\mathrm{OD}_{600}\right)$ of 0,5 . Protein expression was induced with $0.5 \mathrm{mM}$ isopropyl $\beta$-D-thiogalactoside for four hours at $30^{\circ} \mathrm{C}$. Cultures were harvested and the cells lysed by sonication. rFimA was purified by $\mathrm{Ni}$-agarose chromatography (Quiagen) and elution with imidazole. The protein was further purified by a size exclusion on a Superdex 26/60 column (Amersham, Uppsala, Sweden). The rFimA fractions were analyzed by SDS-PAGE and by Western blot.

Western blot of whole cell extracts and recombinant FimA A suspension $(300 \mu \mathrm{ml})$ of bacterial cells $\left(3 \times 10^{9}\right.$ cells $)$ was sonicated $4 \times 15$ seconds using a Branson sonicator (Branson ultrasonics corporation, Danbury, CT). Proteins were precipitated with acetone for 1 hour at $-20^{\circ} \mathrm{C}$, centrifuged and dissolved in $50 \mu \mathrm{ml}$ sample buffer $(62.5 \mathrm{mM}$ Tris, $10.1 \%$ glycerol, $2.0 \%$ SDS, $5 \mathrm{mM}$ dithiothreitol and $0.01 \%$ pyronin) by boiling for $10 \mathrm{~min}$. After centrifuga- 
tion, the supernatant was electrophoretically separated on a 4 to $20 \%$ or 4 to $15 \%$ (whole cell extracts and recombinant FimA, respectively) polyacrylamid gel using Trisglycine buffer (25 mM Tris, $192 \mathrm{mM}$ glycine, and $0.1 \%$ SDS, $\mathrm{pH} 8.3$ ) at $15 \mathrm{~mA}$. The separated proteins were blotted onto membranes (Immobilon PVDF membrane, Millipore Corp., Bedford, MA) using a transblotter. The membranes were blocked with $5 \%$ non-fat dried milk overnight and incubated with antisera R70-3 (diluted $1: 20,000$ ) overnight followed by five washes in $10 \mathrm{mM}$ phosphate buffered saline (PBS), pH 6.8, $0.5 \%$ Tween. Peroxidase conjugated goat anti-rabbit IgG was used as secondary antibody (Dakopatts a/s, Glostrup, Denmark) and a chemiluminescent substrate (Supersignal Substrate; Pierce, Rockford, Il) was used to visualize immobilized antibodies.

\section{Hemagglutination}

Equal volumes (10 $\mu \mathrm{ml}$ of each) of suspensions of bacterial cells $\left(3 \times 10^{9}\right.$ cells $/ \mathrm{ml}$ PBS or reciprocal dilutions) and human, goat or chicken erythrocytes ( $4 \%$ erythrocyte suspension in PBS) were mixed and agitated gently for $1 \mathrm{~min}$ on a glas slide. Erythrocytes were depleted of sialic acid by incubation with 1 unit $/ \mathrm{ml}$ Clostridium perfringens neuraminidase (Sigma Chemical Co, St Louis, MO) for 30 min. at $37^{\circ} \mathrm{C}$. Agglutination was scored visually; $0=$ no agglutination, 1 = weak agglutination, 2 = moderate agglutination, $3=$ strong agglutination, and $4=$ very strong agglutination.

\section{Authors' contributions}

$\mathrm{MD}, \mathrm{KH}$ and UÖ performed laboratory analyses, analysed data and participated in writing the manuscript. $\mathrm{AB}$ sequenced and analysed the fimA gene from PK984 and KP expressed the rFimA protein. IJ and NS designed and planned the project, as well as were responsible for writing the manuscript, at an overall level.

\section{Acknowledgements}

This study was supported by grants from the Swedish Research Council (project 10906 and 200I-3368), the County Council of Västerbotten and from foundations at Umeå University.

\section{References}

I. Hallberg K, Hammarström KJ, Falsen E, Dahlen G, Gibbons RJ, Hay $\mathrm{DI}$, Strömberg N: Actinomyces naeslundii genospecies I and $\mathbf{2}$ express different binding specificities to $\mathrm{N}$-acetyl-b-D-galactosamine, whereas Actinomyces odontolyticus expresses a different binding specificity in colonizing the human mouth. Oral Microbiol Immunol 1998, 13:327-336.

2. Johnson JL, Moore LV, Kaneko B, Moore WE: Actinomyces georgiae sp. nov., Actinomyces gerencseriae sp. nov., designation of two genospecies of Actinomyces naeslundii, and inclusion of A. naeslundii serotypes II and III and Actinomyces viscosus serotype II in A. naeslundii genospecies 2. Int J Syst Bacteriol 1990, 40:273-286.

3. Ellen RP: Establishment and distribution of Actinomyces viscosus and Actinomyces naeslundii in the human oral cavity. Infect Immun 1976, 14: I I 19-1 I24.
4. Mager DL, Ximenez-Fyvie LA, Haffajee AD, Socransky SS: Distribution of selected bacterial species on intraoral surfaces. J Clin Periodontol 2003, 30:644-654.

5. Nyvad B, Kilian M: Microflora associated with experimental root surface caries in humans. Infect Immun 1990, 58: 1628-1633.

6. Becker MR, Paster BJ, Leys EJ, Moeschberger ML, Kenyon SG, Galvin JL, Boches SK, Dewhirst FE, Griffen AL: Molecular analysis of bacterial species associated with childhood caries. J Clin Microbiol 2002, 40: 100I-1009.

7. Tanner A, Maiden MF, Macuch PJ, Murray LL, Kent RLJ: Microbiota of health, gingivitis, and initial periodontitis. J Clin Periodontol 1998, 25:85-98.

8. Raymond A, Smego J, Foglia G: Actinomycosis. Clinical Infectious Diseases 1998, 26: I255-1263.

9. Whittaker CJ, Klier CM, Kolenbrander PE: Mechanisms of adhesion by oral bacteria. Annu Rev Microbiol 1996, 50:513-552.

10. Gibbons RJ: Bacterial adhesion to oral tissues: a model for infectious diseases. J Dent Res 1989, 68:750-760.

II. Kolenbrander PE: Intergeneric coaggregation among human oral bacteria and ecology of dental plaque. Annu Rev Microbiol 1988, 42:627-656.

12. Cisar JO, Sandberg AL, Mergenhagen SE: The function and distribution of different fimbriae on strains of Actinomyces viscosus and Actinomyces naeslundii. J Dent Res 1984, 63:393-396.

13. Cisar JO, Vatter AE, Clark WB, Curl SH, Hurst Calderone S, Sandberg AL: Mutants of Actinomyces viscosus TI4V lacking type I, type 2, or both types of fimbriae. Infect Immun 1988, 56:2984-2989.

14. Hallberg K, Holm C, Öhman U, Strömberg N: Actinomyces naeslundii displays variant fimP and fimA fimbrial subunit genes corresponding to different types of acidic proline-rich protein and beta-linked galactosamine binding specificity. Infect Immun 1998, 66:4403-44I0.

15. Gibbons RJ, Hay DI: Human salivary acidic proline-rich proteins and statherin promote the attachment of Actinomyces viscosus LY7 to apatitic surfaces. Infect Immun 1988, 56:439-445.

16. Stromberg N, Karlsson KA: Characterization of the binding of Actinomyces naeslundii (ATCC I 2 I 04) and Actinomyces viscosus (ATCC 19246) to glycosphingolipids, using a solidphase overlay approach. J Biol Chem 1990, 265: I I 25I-I I 258.

17. Stromberg N, Boren T, Carlen A, Olsson J: Salivary receptors for GalNAc beta-sensitive adherence of Actinomyces spp.: evidence for heterogeneous GaINAc beta and proline-rich protein receptor properties. Infect Immun 1992, 60:3278-3286.

18. Cisar JO, Sandberg AL, Reddy GP, Abeygunawardana C, Bush CA: Structural and antigenic types of cell wall polysaccharides from viridans group streptococci with receptors for oral actinomyces and streptococcal lectins. Infect Immun 1997, 65:5035-504l.

19. Brennan MJ, Cisar JO, Vatter AE, Sandberg AL: Lectin-dependent attachment of Actinomyces naeslundii to receptors on epithelial cells. Infect Immun 1984, 46:459-464.

20. Strömberg N, Borén $\mathrm{T}$ : Actinomyces tissue specificity may depend on differences in receptor specificity for GalNAc beta-containing glycoconjugates. Infect Immun 1992 , 60:3268-3277.

21. Sandberg AL, Ruhl S, Joralmon RA, Brennan MJ, Sutphin MJ, Cisar JO: Putative glycoprotein and glycolipid polymorphonuclear leukocyte receptors for the Actinomyces naeslundii WVU45 fimbrial lectin. Infect Immun 1995, 63:2625-263I.

22. Yeung MK: Conservation of an Actinomyces viscosus TI4V type I fimbrial subunit homolog among divergent groups of Actinomyces spp. Infect Immun 1992, 60:1047-1054.

23. Yeung MK, Chassy BM, Cisar JO: Cloning and expression of a type I fimbrial subunit of Actinomyces viscosus TI4V. J Bacteriol 1987, 169:1678-1683.

24. Yeung MK, Cisar JO: Cloning and nucleotide sequence of a gene for Actinomyces naeslundii WVU45 type 2 fimbriae. J Bacteriol 1988, 170:3803-3809.

25. Yeung MK, Cisar JO: Sequence homology between the subunits of two immunologically and functionally distinct types of fimbriae of Actinomyces spp. J Bacteriol 1990, 172:2462-2468.

26. Yeung MK, Ragsdale PA: Synthesis and function of Actinomyces naeslundii TI4V type I fimbriae require the expression of 
additional fimbria-associated genes. Infect Immun 1997, 65:2629-2639.

27. Li T, Khah MK, Slavnic S, Johansson I, Strömberg N: Different type I fimbrial genes and tropisms of commensal and potentially pathogenic Actinomyces spp. with different salivary acidic proline-rich protein and statherin ligand specificities. Infect Immun 200I, 69:7224-7233.

28. Loimaranta V, Jakubovics NS, Hytonen J, Finne J, Jenkinson HF, Strömberg N: Fluid- or surface-phase human salivary scavenger protein gp340 exposes different bacterial recognition properties. Infect Immun 2005, 73:2245-2252.

29. Ton-That H, Marraffini LA, Schneewind O: Sortases and pilin elements involved in pilus assembly of Corynebacterium diphtheriae. Mol Microbiol 2004, 53:25I-26I.

30. Ton-That $\mathrm{H}$, Schneewind $\mathrm{O}$ : Assembly of pili on the surface of Corynebacterium diphtheriae. Mol Microbiol 2003, 50:1429-1438.

31. Ton-That H, Marraffini LA, Schneewind O: Protein sorting to the cell wall envelope of Gram-positive bacteria. Biochim Biophys Acta 2004, 1694:269-278.

32. van Die I, Hoekstra W, Bergmans $\mathrm{H}$ : Analysis of the primary structure of P-fimbrillins of uropathogenic Escherichia coli. Microb Pathog 1987, 3:149-154.

33. Yeung MK: Complete nucleotide sequence of the Actinomyces viscosus TI4V sialidase gene: presence of a conserved repeating sequence among strains of Actinomyces spp. Infect Immun 1993, 61:109-116.

34. Molecular Toolkit [http://arbl.cvmbs.colostate.edu/molkit/ index.html]. .

35. Genome Net, ClustalW [http://clustalw.genome.jp/].

36. Center for Biological Sequence analysis, SignalP server [http://www.cbs.dtu.dk/services/SignalP/].

37. ExPASy Proteomics Server, ScanProsite [http://
[ www.expasy.org/tools/scanprosite/].

38. My hits, motif scan [http://myhits.isb-sib.ch/cgi-bin/ motif_scan].

39. Navarre WW, Schneewind O: Proteolytic cleavage and cell wall anchoring at the LPXTG motif of surface proteins in grampositive bacteria. Mol Microbiol 1994, I4:1 I5-121.

Publish with Bio Med Central and every scientist can read your work free of charge

"BioMed Central will be the most significant development for disseminating the results of biomedical research in our lifetime. "

Sir Paul Nurse, Cancer Research UK

Your research papers will be:

- available free of charge to the entire biomedical community

- peer reviewed and published immediately upon acceptance

- cited in PubMed and archived on PubMed Central

- yours - you keep the copyright 